\title{
Preventation of Data Content Leakage with Secured Encryption Algorithm
}

\author{
Mr. Sagar Prasad', Ms. Malti Nagle ${ }^{2}$, Mr. Tarique Zeya Khan ${ }^{3}$ \\ Research Scholar M Tech, Comp Sci. \& Eng, Surabhi College of Eng \& Tech, Bhopal, M P, India ${ }^{1}$ \\ Asst. Prof., M Tech, Comp Sci. \& Eng, Surabhi College of Eng \& Tech, Bhopal, M P, India ${ }^{2,3}$
}

\begin{abstract}
The information leak of sensitive data on systems has a serious threat to organization data security. Statistics show that the improper encryption on files and communications due to human errors is one of the leading causes of information loss. So there a need tools to identify the exposure of sensitive data by monitoring the content in storage and transmission. However, detecting the exposure of sensitive data information is challenging due to data transformation in the content. Transformations result in highly unpredictable leak patterns. In this paper, it is utilize sequence alignment techniques used for detecting complex data-leak patterns. This algorithm is designed for detecting long and inexact sensitive data patterns. This detection is paired with a comparable sampling algorithm, which allows one to compare the similarity of two separately sampled sequences. The system achieves good detection accuracy in recognizing transformed leaks. It implement a parallelized version of our algorithms in graphics processing unit to achieves high analysis data. In the case of collective privacy preservation, organizations have to cope with some interesting conflicts. For instance, when personal information undergoes analysis processes that produce new facts about users' shopping patterns, hobbies, or preferences, these facts could be used in recommender systems to predict or affect their future shopping patterns. In general, this scenario is beneficial to both users and organizations. However, when organizations share data in a collaborative project, the goal is not only to protect personally identifiable information but also sensitive knowledge represented by some strategic patterns.
\end{abstract}

Keywords: Information leak detection, content inspection, sampling, alignment, dynamic programming.

\section{INTRODUCTION}

To Protect the exposure of sensitive data and documents, an organization needs to prevent text sensitive data from appearing in the storage or communication. In today's increasingly digital world, there is often a tension between safeguarding privacy and sharing information. Although, in general, sensitive data clearly needs to be kept confidential, data owners are often motivated, or forced, to share sensitive information Privacy-Preserving Sharing of Sensitive Information, and proposes one efficient and secure instantiation that functions as a privacy shield to protect parties from disclosing more than the required minimum of sensitive information.

We model in the context of simple database-querying applications with two parties: a server that has a database, and a client, performing simple disjunctive equality queries Detecting the exposure of sensitive information is challenging due to data transformation in the content. Transformations result in highly unpredictable leak patterns. In this paper, we utilize sequence alignment techniques for detecting complex data-leak asymmetric cryptography, facilitate the creation of a verifiable association between a public key and the identity other attributes of the holder of the corresponding private key, for uses such as authenticating the identity of a specific entity, ensuring the integrity of information, providing support for non repudiation, and establishing an encrypted communications section.

\section{PROPOSED SYSTEM}

1) Key Authorities: They are key generation centers that generate public/secret parameters for. The key authorities consist of a central authority and multiple local authorities. We assume that there are secure and reliable communication channels between a central authority and each local authority during the initial key setup and generation phase.

Each local authority manages different attributes and issues corresponding attribute keys to users.

They grant differential access rights to individual users based on the users' attributes. The key authorities are assumed to be honest-but-curious. That is, they will honestly execute the assigned tasks in the system, however they would like to learn information of encrypted contents as much as possible.

2) Storage node: This is an entity that stores data from senders and provide corresponding access to users. It may be mobile or static. Similar to the previous schemes, we also assume the storage node to be semitrusted, that is honest-but-curious.

3) Sender: This is an entity who owns confidential messages or data (e.g., a commander) and wishes to store them into the external data storage node for ease of sharing or for reliable delivery to users in the extreme networking environments. A sender is responsible for defining (attribute based) access policy and enforcing it on 
its own data by encrypting the data under the policy before storing it to the storage node.

4) User: This is a mobile node who wants to access the data stored at the storage node (e.g., a soldier). If a user possesses a set of attributes satisfying the access policy of the encrypted data defined by the sender, and is not revoked in any of the attributes, then he will be able to decrypt the DES ALGORTHIM and obtain the data. Since the key authorities are semi-trusted, they should be deterred from accessing plaintext of the data in the storage node; they should be still able to issue secret keys to users.

\section{Pervious Algorithm CPABE}

We propose an attribute-based secure data retrieval scheme using CP-ABE for decentralized DTNs. The proposed scheme features the following achievements. First, immediate attribute revocation enhances backward/forward secrecy of confidential data by reducing the windows of vulnerability. Second, encryptors can define a fine-grained access policy using any monotone access structure under attributes issued from any chosen set of authorities. Third, the key escrow problem is resolved by an escrow-free key issuing protocol that exploits the characteristic of the decentralized DTN architecture. The key issuing protocol generates and issues user secret keys by performing a secure two-party computation (2PC) protocol among the key authorities with their own master secrets. The $2 \mathrm{PC}$ protocol deters the key authorities from obtaining any master secret information of each other such that none of them could generate the whole set of user keys alone.

Thus, users are not required to fully trust the authorities in order to protect their data to be shared. The data confidentiality and privacy can be cryptographically enforced against any curious key authorities or data storage nodes in the proposed scheme.

\section{DES WITH MD5 ALGORTHIM}

3DES encrypts a 64-bit block of plaintext to 64-bit block of ciphertext. It usesa 128-bit key. The algorithm consists of eight identical rounds and a "half" roundfinal Transformation.

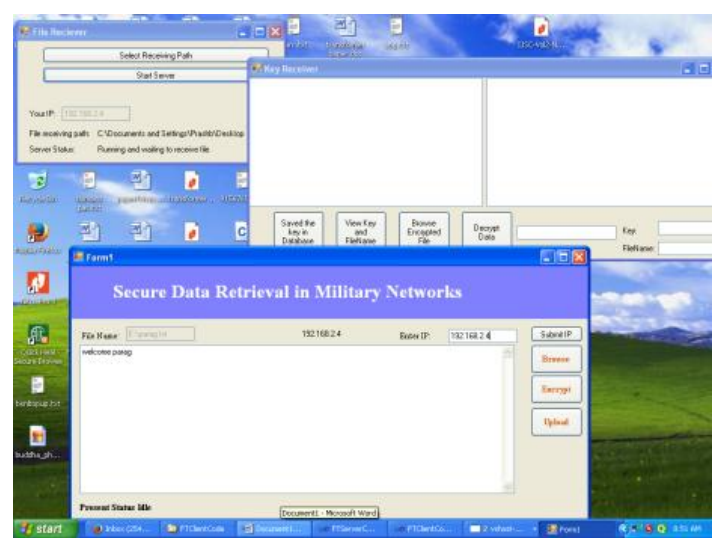

Fig 1. Secured Data retrieval system
There are 216 possible 16-bit blocks: Each operation with the set of possible 16-bit blocks is an algebraic group. Bitwise XOR is bitwise addition modulo 2,and addition modulo 216 is the usual group operation. Some spin must be put on the elements - the 16-bit blocks - to make sense of multiplication modulo $216+1$, however. 0 is not an element of the multiplicative group.

Confidentiality: In order to protect sensed data and communication exchanges between sensor nodes it is important to guarantee the secrecy of messages. In the sensor network case this is usually achieved by the use of symmetric cryptography as asymmetric or public key cryptography in general is considered too expensive. However, while encryption protects against outside attacks, it does not protect against inside attacks/node compromises, as an attacker can use recovered cryptographic key material to successfully eavesdrop, impersonate or participate in the secret communications of the network. Furthermore, while confidentiality guarantees the security of communications inside the network it does not prevent the misuse of information reaching the base station. Hence, confidentiality must also be coupled with the right control policies so that only authorized users can have access to confidential information.

Integrity and Authentication: authentication is necessary to enable sensor nodes to detect modified, injected, or replayed packets. While it is clear that safety-critical applications require authentication, it is still wise to use it even for the rest of applications since otherwise the owner of the sensor network may get the wrong picture of the sensed world thus making inappropriate decisions. However, authentication alone does not solve the problem of node takeovers as compromised nodes can still authenticate themselves to the network. Hence authentication mechanisms should be "collective" and aim at securing the entire network.

In particular, the following requirements must be supported by the key management scheme, in order to facilitate data aggregation and dissemination process: First we focused on the establishment of trust relationship among wireless sensor nodes, and presented a key management protocol for sensor networks. The protocol includes support for establishing four types of keys per sensor node: individual keys shared with the base station, pairwise keys shared with individual neighboring nodes, cluster keys shared with a set of neighbors, and a group key shared with all the nodes in the network.

We showed how the keys can be distributed so that the protocol can support in-network processing and efficient dissemination, while restricting the security impact of a node compromise to the immediate network neighborhood of the compromised node. Applying the protocol makes it really hard for an adversary to disrupt the normal operation of the network. 


\section{IV.RESULT}

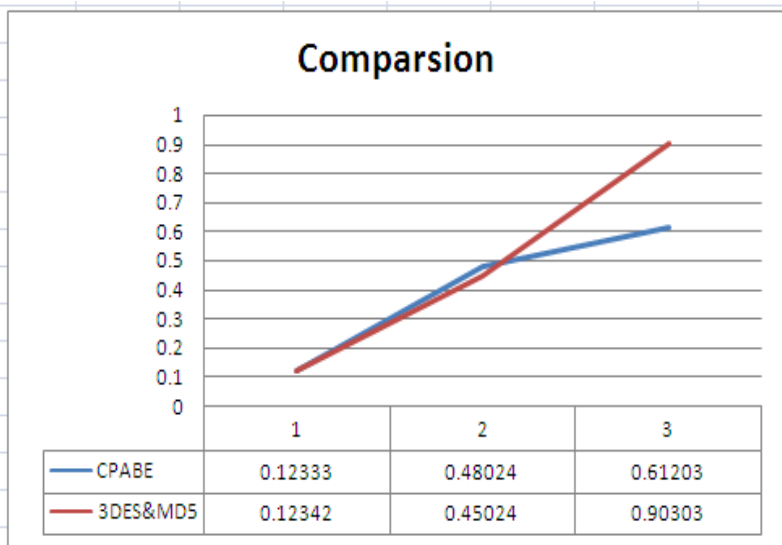

Fig 2. Packet Delivery Ratio

\section{CONCLUSION}

The corresponding attribute group keys are updated and delivered to the valid attribute group members securely (including the user). In addition, all of the components encrypted with a secret key in the ciphertext are reencrypted by the storage node with a random, and the ciphertext components corresponding to the attributes are also reencrypted with the updated attribute group keys. Even if the user has stored the previous ciphertext exchanged before he obtains the attribute keys and the holding attributes satisfy the access policy, he cannot decrypt the pervious ciphertext.

\section{REFERENCES}

[1] Hiroki Nishiyama, Desmond Fomo, Zubair Md. Fadlullah,, and NeiKato,Fellow," Traffic Pattern Based Content Leakage Detection for Trusted Content Delivery Networks" IEEE Transaction on Parallel and Distributed System, Volume 25, No 2 Feb 2014

[2] K. Ramya, D. RamyaDorai, Dr. M. Rajaram "Tracing Illegal Redistributors of Streaming Contents using Traffic Patterns" IJC A 2011

[3] A. Asano, H. Nishiyama, and N. Kato, "The Effect of Packet Reordering and Encrypted Traffic on Streaming Content Leakage Detection" Proc. Int'l Conf. Computer Comm. Networks (ICCCN '10), pp. 1 6, Aug. 2010.

[4] Y. Chu, S.G. Rao, S. Seshan, and H. Zhang, "Enabling Conferencing Applications on the Internet Using an Overlay Multicast Architecture,” Proc.ACM SIGCOMM, pp. 55 67,Aug. 2010

[5] O. Adeyinka, "Analysis of IPSec VPNs Performance in a Multimedia Environment," Proc. Fourth Int'l Conf. Intell igent Environments, pp. 25 - 30, 2008

[6] M. Dobashi, H. Nakayama, N. Kato, Y. Nemoto, and A. Jamalipour, "Traitor Tracing Technology of Streaming Contents Delivery Using Traffic Pattern in Wired/Wireless Environments," Proc. IEEE Global Telecomm. Conf., pp. 1 5, Nov./Dec. 2006.

[7] S. Amarasing and M. Lertwatechakul, "The Study of Streaming Traffic Behavior," KKU Eng. J., vol. 33, no. 5, pp. 541 553, Sept./Oct. 2006.

[8] R.S. Naini and Y. Wang, "Sequential Traitor Tracing," IEEE Trans. Information Theory, vol. 49, no. 5, pp. 1319 1326, May 2003.

[9] D. Geiger, A. Gupta, L.A. Costa, and J. Vlontzos, "Dynamic Programming for Detecting, Tracking, and Matching Deformable C ontours," Proc. IEEE Trans. Pattern Analysis and Machine Intelligence, vol. 17, no. 3, pp. 294 302, M ar. 1995.

\section{BIOGRAPHIES}

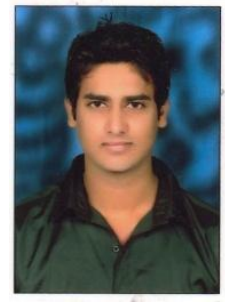

Sagar Prasad received the bachelor's degree in computer science and engineering from SBITM college from RGPV University of Bhopal in 2014.He is currently Pursuing the M.Tech in computer science and engineering from SCET college From RGPV University Bhopal, M.P.

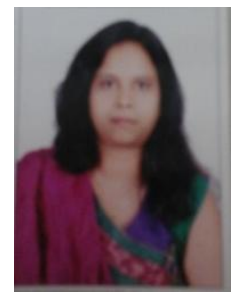

Malti Nagle received the B.E.in Information Technoloy from Samrat Ashok Technologyical Institute VIDISHA (M.P)in 2006 and M.tech in Computer Science \& Engineering from Jaypee University of Information Technology(U.P)in 2009. She is a professor of Computer Science \& Engg With Surabhi College Of Enginerring BHOPAL, MP. Prior to that, she led the IT Trainer At Smritinet.com. BHOPAL, MP. Her main research intrests are Network security and ADHOC Network in which she has published more than 15 papers. Prof Malti was an IEEE review committee member at SoftCOM 2014.she has been in education profession from 7years. She worked in various university as A.P. 